\title{
RESULTS FROM SEARCHES FOR HIGH-REDSHIFT GALAXIES AROUND DAMPED LY $\alpha$ SYSTEMS
}

\author{
S. J. WARREN \\ Astrophysics, Blackett Laboratory \\ ICSTM, Prince Consort Rd, London, SW7 2BZ, UK \\ AND \\ P. MØLLER \\ Space Telescope Science Institute, \\ 3700 San Martin Drive, Baltimore, MD 21218, USA \\ on assignment from the Space Science Department of ESA
}

\section{Introduction}

There was not much said at this conference about the damped Ly $\alpha$ absorbers, yet locally and at high redshift nearly all the neutral hydrogen in the Universe lies in the damped systems, so they may have much to tell us about when and how galaxies formed. This talk is concerned with the results of narrow-band imaging searches for Ly $\alpha$ emission from, and around, damped Ly $\alpha$ absorbers. There have been many such searches, but very few detections. Here we concentrate on two groups of galaxies at high redshift, $z>2$, found by this technique. One of these is a recent discovery (Francis et al. 1995), and for the other we discuss some new observations (Warren and Møller 1995). A study of these two groups provides some clues to the nature of the damped absorbers at high redshift, and the rôle they play in the formation of galaxies.

\section{Damped Ly $\alpha$ absorbers}

The damped systems form the high-column end of the distribution of Ly $\alpha$ absorbers, $\mathrm{N}(\mathrm{HI})>10^{20} \mathrm{~cm}^{-2}$. The shape of the column-density distribution means that virtually all the neutral gas in the Universe is contained in the damped systems, so surveys for damped absorbers in the spectra of quasars can be used to measure the cosmic density of neutral gas $\Omega_{\text {DLAA }}$. 
Lanzetta et al. (1995) provide a plot of $\Omega_{\mathrm{DLA}}$ in the redshift range $0<z<3$. There is a rough correspondence between the mass in neutral gas at $z=3$, and the mass in stars in spiral galaxy disks today, and the standard picture has been that the damped systems at $z=3$ are the fully formed gaseous disks of spiral galaxies, that turn into the stellar disks of today. The disks would have been a factor $>2$ larger in the past, in order to explain the large number of absorbers found. However, the mass in stars today is not known to better than a factor of 2 , and given the low metallicity of the damped systems at $z>2$, we could instead be seeing the gas from which all stars formed, i.e. in both disks and spheroids. Nevertheless, accounting for the effects of dust in the damped systems may be critical in such considerations (Pei and Fall 1995). This needs to be quantified better before drawing firm conclusions about the history of star formation in the damped absorbers.

\section{Ly $\alpha$ searches}

The theme of this talk is the comparison between what we observe at high redshift, and the predictions of hierarchical clustering theories (Frenk, this volume). In the simulations the constituents of a galaxy today reside at $z=3$ in a small number of pieces, which can lie far apart. A nice illustration of this is given by Navarro et al. (1995). In their Fig. 1 we see at $z=3$ two large clumps of cold gas separated by a proper distance of $100 \mathrm{~h}^{-1} \mathrm{kpc}$, that merge at $z \sim 1$ to form a disk galaxy. Therefore in searching for emission around damped systems at $2<z<3$ we should be thinking in terms of sub-units of galaxies at angular separations from the line of sight to the quasar of, say, $30 \operatorname{arcsec}\left(1 \operatorname{arcsec} \equiv \sim 4 h^{-1} \mathrm{kpc}, 2<z<3, q_{\mathrm{o}}=0.5\right)$.

\subsection{A GROUP OF GALAXIES AT $Z=2.38$ TOWARDS THE QUASAR $2139-4434$}

This group was discovered at the end of 1994. Francis and Hewett (1993) describe the spectra of two high-z quasars separated by 8 arcmin, in which in each spectrum common high-column-density absorption is seen at each of two redshifts, $z=2.38, z=2.85$. This common absorption may be due to a galaxy supercluster, and follow-up narrow-band imaging in Ly $\alpha$ has begun in an effort to map out the structure. Francis et al. (1995) describe the results from imaging towards the quasar 2139-4434, targeted at the absorber at $z=2.38$. This absorber has a column density $7.0 \times 10^{18} \mathrm{~cm}^{-2}$, so in fact it is a Ly-limit system rather than a damped system. The narrowband image revealed two candidate high-redshift galaxies, and subsequent spectroscopy has confirmed Ly $\alpha$ emission from each. The brighter source, $f=8 \times 10^{-16} \mathrm{erg} \mathrm{s}^{-1} \mathrm{~cm}^{-2}$, lies at 22 arcsec from the line of sight to the quasar. It is extended over some 5 arcsec, and probably shows Civ 
emission. In many respects it resembles the high-redshift radio galaxies, although it is radio quiet. The second source, $f=3 \times 10^{-16} \mathrm{erg} \mathrm{s}^{-1} \mathrm{~cm}^{-2}$, lies at 63 arcsec separation. The two galaxies are certainly bright enough to have been discovered in the wide-field blank-sky surveys by Thompson and Djorgovski, had they lain in one of their fields.

There are three objects at $z=2.38$ in this field: the Ly $\alpha$ absorber, and the two emission-line galaxies. The main feature that we draw attention to is the fact that the three sources are closely aligned. We return to this point below.

\subsection{EMISSION-LINE GALAXIES AT $Z=2.81$ TOWARDS PKS0528-250}

The second absorber we discuss is the unusual damped system at $\mathrm{z}=2.81$ seen in the spectrum of the quasar PKS0528-250. The absorber lies suffciently close that the quasar's Ly $\alpha$ emission line is completely absorbed. Moller and Warren (1993) imaged the system at Ly $\alpha$, in an attempt to measure the size of the absorber. The idea was that the quasar would light up the backside of the absorber, and the periphery of the cloud would be visible as a ring of $\mathrm{Ly} \alpha$ emission. However the narrow-band image did not reveal a ring. Rather three blobs of emission were found at angular separations of $1.2,11$, and 21 arcsec from the quasar.

Subsequent spectroscopy and deep imaging of this field are presented in Warren and Møller (1995). The interpretation of what we are seeing here is not straightforward. Briefly, based on the morphology of the three blobs, the line velocity widths, and the line equivalent widths, we are led to the conclusion that we are seeing star formation rather than photoionisation by the quasar. As with the field studied by Francis et al. the three sources are closely aligned. One possibility is that they are three regions of star formation in an edge on disk. They span a distance of $80 h^{-1} \mathrm{kpc}$, and this would accord with the picture outlined above of spiral galaxy disks being larger in the past. However the velocities are inconsistent with systematic rotation: the middle blob is redshifted with respect to the outer two blobs. Therefore we seek an alternative explanation.

We put forward the idea that the alignments of galaxies seen in these two fields correspond to the filamentary structures found in simulations of hierarchical theories of galaxy formation: See e.g. Evrard, Summers, and Davis (1994) for good plots showing this phenomenon. At this conference Windhorst et al. presented a third group of high-redshift galaxies. There are four objects which apparently show emission lines in a narrow-band image. Three of these (ohjects A, B, R) have redshifts confirmed by spectroscopy, and once again they are closely aligned. We predict that such alignments will prove common, as more high-redshift galaxies are discovered. 
The mass of the gas in the 0528 absorber is estimated to be $1 \times 10^{9} h^{-2} \mathrm{M}_{\odot}$, and the total mass $4 \times 10^{10} h^{-1} \mathrm{M}_{\odot}$. The dynamical mass of the group is $\sim 3 \times 10^{11} h^{-1} \mathrm{M}_{\odot}$, and the dynamical time a few times $10^{8}$ yrs. The group resembles groups seen in the computer simulations, that coallesce at lower redshift. The overall picture that emerges is of star formation in a sub- $\mathrm{L}_{\star}$ galaxy, at an early stage in its evolution, during the process of assembly.

\section{Remarks on the nature of galaxies at high redshift $z>2$}

1. The sensitivity of emission-line searches for high-z galaxies may be compromised by the fact that today's galaxies are in pieces at high redshift.

2. There are three confirmed groups of galaxies at $2<z<3$. All show alignment, which may correspond to the filamentary structures seen in simulations of galaxy formation.

3. At $z \sim 3$ at least some of the damped systems are probably subcomponents of galaxies in the process of assembly, rather than the fullyformed progenitors of spiral-galaxy disks.

\section{References}

Evrard, A. E., Summers, F. J. and Davis, M. (1994) Ap.J 422, 11

Francis, P. J. and Hewett, P. C. (1993) A.J 105, 1633

Francis, P. J., Woodgate, B. E., Warren, S. J., et al. (1995) ApJ in press

Lanzetta, K. M., Wolfe, A. M. and Turnshek, D. A. (1995) A p.J 440, 435

Møller, P. and Warren, S. J. (1993) A\&A 270, 43

Navarro, J. F., Frenk, C. S. and White, S. D. M. (1995) MNRAS 275, 56

Pei, Y. C. and Fall, S. M. (1995) ApJ in press

Warren, S. J. and Møller, P. (1995) A\&A in press

\section{DISCUSSION:}

Fritze-v. Alvensleben: Is the COBE upper limit on dust in agrecment with estimates of dust in the damped systems, made from measurements of the reddening of QSOs, and from the $\mathrm{Cr}$ to $\mathrm{Zn}$ ratio?

Warren: Yes. The dust-to-gas ratio in the damped systems at $2<z<3$ is only about $1 / 10$ th of that in the local group, but this would be sufficient to extinguish Ly $\alpha$ effectively, if resonant scattering is important.

Rees: The aligned emission regions are slightly reminiscent of what is seen in high-z radio sources. Do you think there is any relation?

Warren: I suppose it is just possible, since two of the fields contain radio sources. None of the three aligned objects in the Francis field is a radio source, however.

Windhorst: In the case of our group at $z=2.40$, the other Ly $\alpha$ emitters (objects $\mathrm{A}$ and $\mathrm{B}$ ) occur in a direction $\sim 40^{\circ}$ different from the radio axis of 5.3W002 (object $R$ ). 